\title{
InSAR in the clouds: satellite-based monitoring at Grasberg Mine
}

\author{
JM Leighton 3vGeomatics Inc., Canada \\ M Sullivan Freeport McMoRan, Indonesia
}

\begin{abstract}
Grasberg mining operations, operated by PT Freeport Indonesia (PTFI), offers unique challenges for monitoring ground displacement using satellite-based Interferometric Synthetic Aperture Radar (InSAR). The conditions on the ground, the extreme terrain and the turbulent tropical atmosphere all present problems that must be overcome to extract meaningful information about where, how much and when the ground is moving. This information provides a big picture overview for tracking geohazards, either in areas where no other monitoring exists or to inform decisions regarding ground instrumentation.

InSAR is a wide-area monitoring technology that can generate over 10,000 ground displacement measurements per square kilometre with sub-centimetre precision. Although InSAR is a mature technology for monitoring ground instabilities, it has been put to the test in this unique environment since InSAR monitoring of the site began in 2014. Images are acquired every 11 days from two different directions to build a history of ground displacement. Despite the challenges at Grasberg, InSAR processing has been optimised with site-specific improvements to enable near-real-time displacement monitoring. The monitoring program encompasses the entire mine site including the pit, stockpiles, infrastructure, access roads and residential communities. In addition, specific tools were developed for monitoring surface displacement caused by the block caving operations.

The operational monitoring program now deployed at Grasberg enhances the early warning systems for ongoing risk management. It has been integrated into mine operations through the monitoring control centres for the pit, underground, and civil geotechnical groups.
\end{abstract}

Keywords: InSAR, monitoring, remote sensing, displacement

\section{$1 \quad$ Introduction}

Grasberg mining operations is located in Papua, Indonesia. The operations span more than $120 \mathrm{~km}$ from the coastal port of Amamapare located at sea level to the Grasberg pit located 4,200 $\mathrm{m}$ above sea level, near the Carstensz Glacier. The dense equatorial rain forest gently rises until $50 \mathrm{~km}$ inland where the terrain dramatically changes to a mountainous region with dramatic vertical relief, climbing $2,000 \mathrm{~m}$ in the next $18 \mathrm{~km}$ and an additional $2,000 \mathrm{~m}$ in the final $8 \mathrm{~km}$. Figure 1 provides an overview of the entire PT Freeport Indonesia (PTFI) property and the respective areas within. These include the mine itself and the approach roads leading up to the mine, termed the Civil Geotech area. This paper focuses on displacement monitoring at Grasberg mine only.

These steep slopes remain constantly wet due to high precipitation in excess of $5 \mathrm{~m}$ per annum in some locations. Because of the high precipitation rates and steep slopes, instabilities along the access roads connecting the mining town of Tembagapura to the lowlands and the Grasberg mine are a reality that must be managed. In addition to the instabilities along the only access road that serves the area, there is a need to monitor the stability of the mining operations in the open pit area and the progression of the surface subsidence from the block caving areas. 


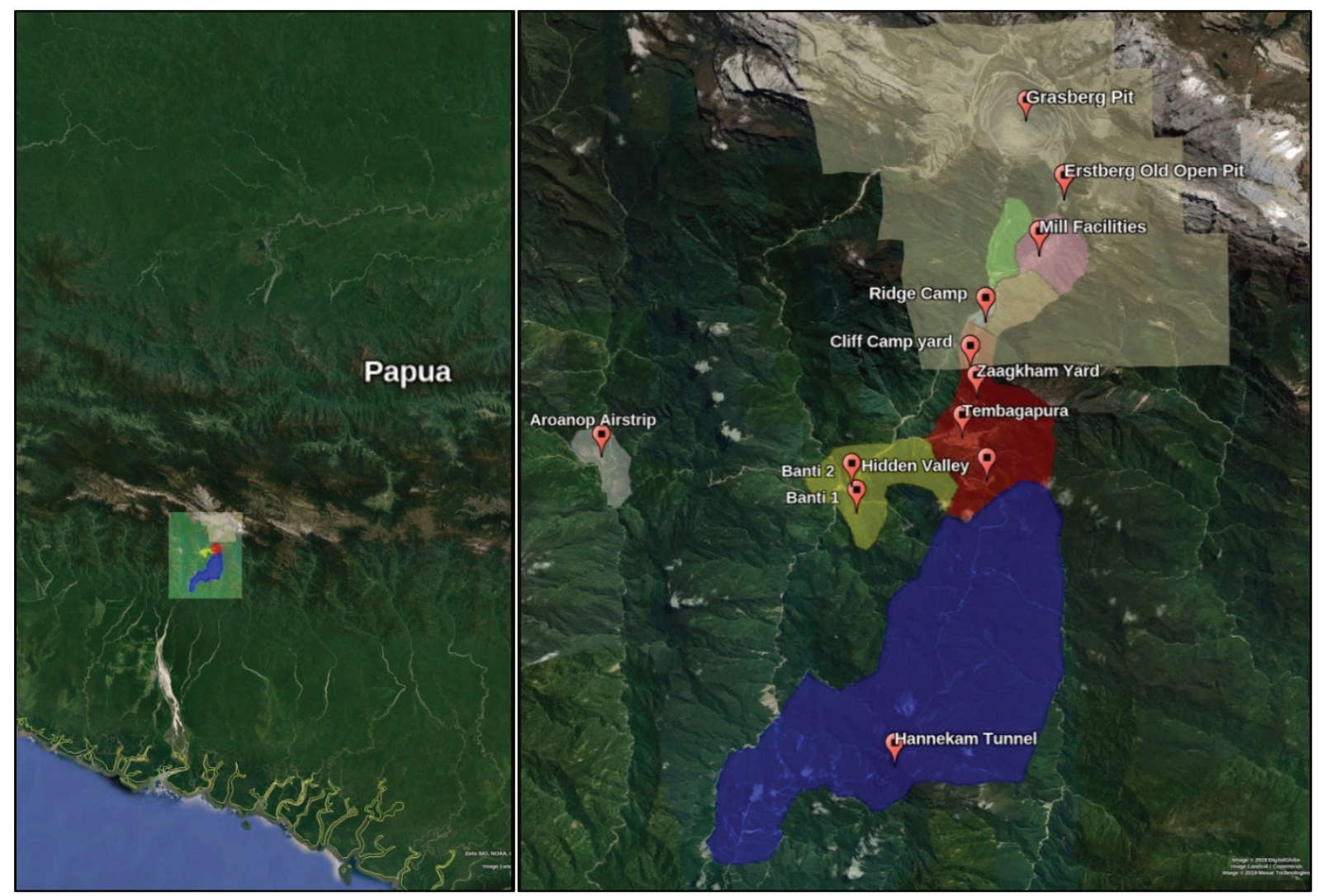

(a)

(b)

Figure 1 (a) An overview of the PT Freeport Indonesia (PTFI) property in Papua; (b) Grasberg Pit and the Civil Geotech area

\section{Methodology}

\subsection{InSAR monitoring}

Interferometric synthetic aperture radar (InSAR) is a processing technique applied to two or more synthetic aperture radar (SAR) images of the same area, to generate ground displacement (Hooper et al. 2004; Massonnet et al. 1993). The two radar images form an interferogram, which represents the per-pixel phase difference between the images, which encompass the changes that have occurred between the two dates. Interferograms contain a number of contributions from noise and error sources, intermixed with ground displacement signal. The essence of InSAR processing therefore is to remove the unwanted contributions, revealing the ground displacement.

Figure 2 shows the evolution of an interferogram: Two SAR images are acquired, dated 20 June and 11 July 2016 (shown on the left as amplitude images) and an interferogram is formed. A simplified narrative of the displacement now follows, numbered in the figure and below:

1. The topographic signal is removed, by subtracting a digital elevation model (DEM).

2. Long scale atmospheric effects caused by water vapour are estimated and subtracted.

3. Height dependent atmospheric effects caused by pressure variations are subtracted.

4. Filtering enhances coherent areas, and reduces noise.

5. Leftover artefacts from the DEM are estimated and removed by analysing a set of interferograms. The resulting phase measurements are now almost completely due to ground displacement.

6. A displacement rate is estimated from the set of interferograms, using linear regression. 


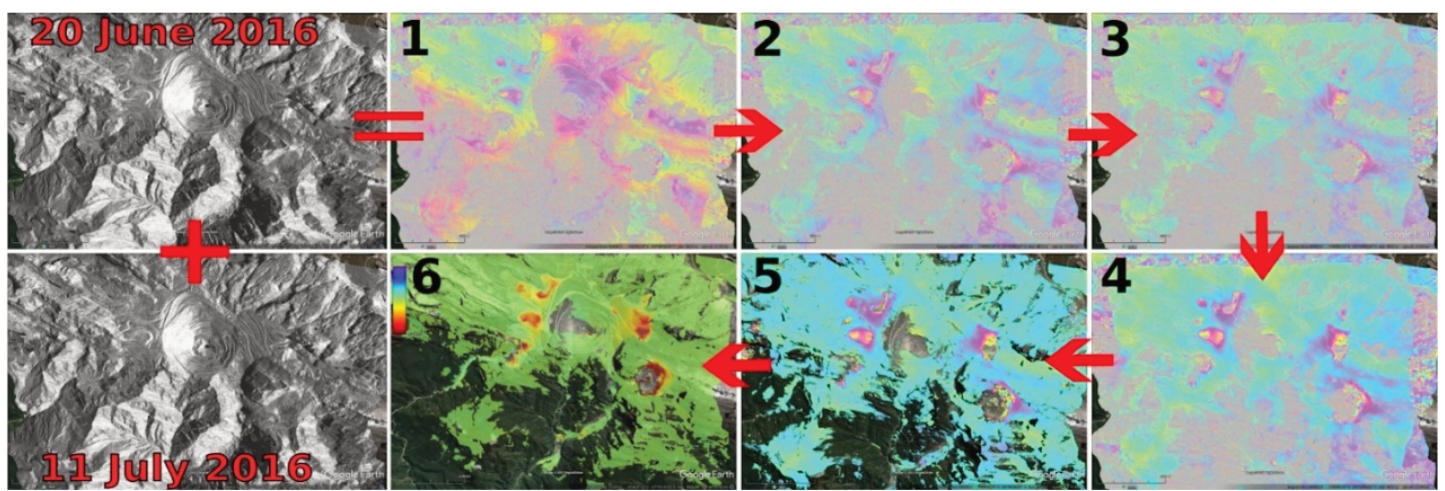

Figure 2 The evolution of synthetic aperture radar (SAR) data from two images acquired on 20 June and 11 July 2016. Successive stages remove unwanted errors and biases, and enhance displacement signal

\subsection{Limitations}

Some limitations of the technology are discussed in the following subsections.

\subsubsection{Satellite geometry}

InSAR estimates ground displacement along the satellite line of sight (LOS) (European Space Agency 2014). If the ground displacement is measured by overlapping ascending and descending passes, it is possible to fuse these measurements in order to derive the direction of displacement within an up-down-east-west plane. Note that InSAR is not suitable for estimating horizontal displacement in the north-south direction.

\subsubsection{Coherence loss}

Excavation and dumping destroys the InSAR phase measurements in mining areas, this is properly known as a loss in coherence (European Space Agency 2014), but this only affects the area being disturbed and only during the period of activity. For example, InSAR measurements formed from images captured on the first and second date may suffer coherence loss in areas that were disturbed during that period, but measurements may return in subsequent image pairs as excavation etc., moves on to other areas of the mine. The resulting analyses, from data with gaps in time, produces time series with gaps in time, which are nevertheless functional and useful, as long as periods of disturbance do not dominate.

Other common causes for reduced coherence are high soil moisture content, vegetation and high magnitude of displacement. This latter case occurs in the Deep Ore Zone (DOZ) area, and is analogous to contours of displacement densifying to the point where they lie on top of one another.

\section{Data}

\subsection{InSAR monitoring at PT Freeport Indonesia}

The SAR data used for monitoring PTFI is acquired by the German TerraSAR-X Satellite, which has a resolution of $3 \mathrm{~m}$, a footprint of $30 \mathrm{~km} \times 50 \mathrm{~km}$, and a revisit time of 11 days. In spite of InSAR challenges at PTFI, the technology has proved to be a powerful risk monitoring technique. To achieve this, many non-standard steps have been developed to overcome the unique nature of the terrain and the environment. Three of the main challenges that were overcome involve:

1. Extreme topography and parallax.

2. Extreme topography and air pressure.

3. High displacement magnitudes.

These topics are now discussed in more detail, highlighting the problem, the solution, and the end results. 


\subsubsection{Extreme topography}

The topographic relief at PTFI has two effects, the first involves image parallax. Consider that the two images that form an interferogram, are acquired from slightly different viewpoints, separated by some distance (typically around 0 to $500 \mathrm{~m}$ ) termed the baseline. Prior to InSAR processing, the images must be mutually aligned at the sub-pixel level, this is called coregistration. But this process is further complicated in areas of highly varying topography, such as the highlands at PTFI. When baselines are large, the viewpoints become less and less similar.

For PTFI, standard image alignment, or coregistration, is first performed using the orbit information and image matching algorithms. After this, the process departs from standard InSAR and the DEM is used to estimate the topographically induced effects between pixels of the two scenes (Nitti et al. 2011), after which the initial coregistration is iterated.

The resulting improvement in signal quality, especially for large baseline interferograms, can be impressive, seemingly revealing signal where only noise existed before. Figure 3 shows sections of the same interferogram without (left) and with (right) the extra coregistration steps using the DEM. The reduction in noise in the image on the right is purely a function of correctly aligning the respective viewpoints for the two SAR images that form the interferogram.

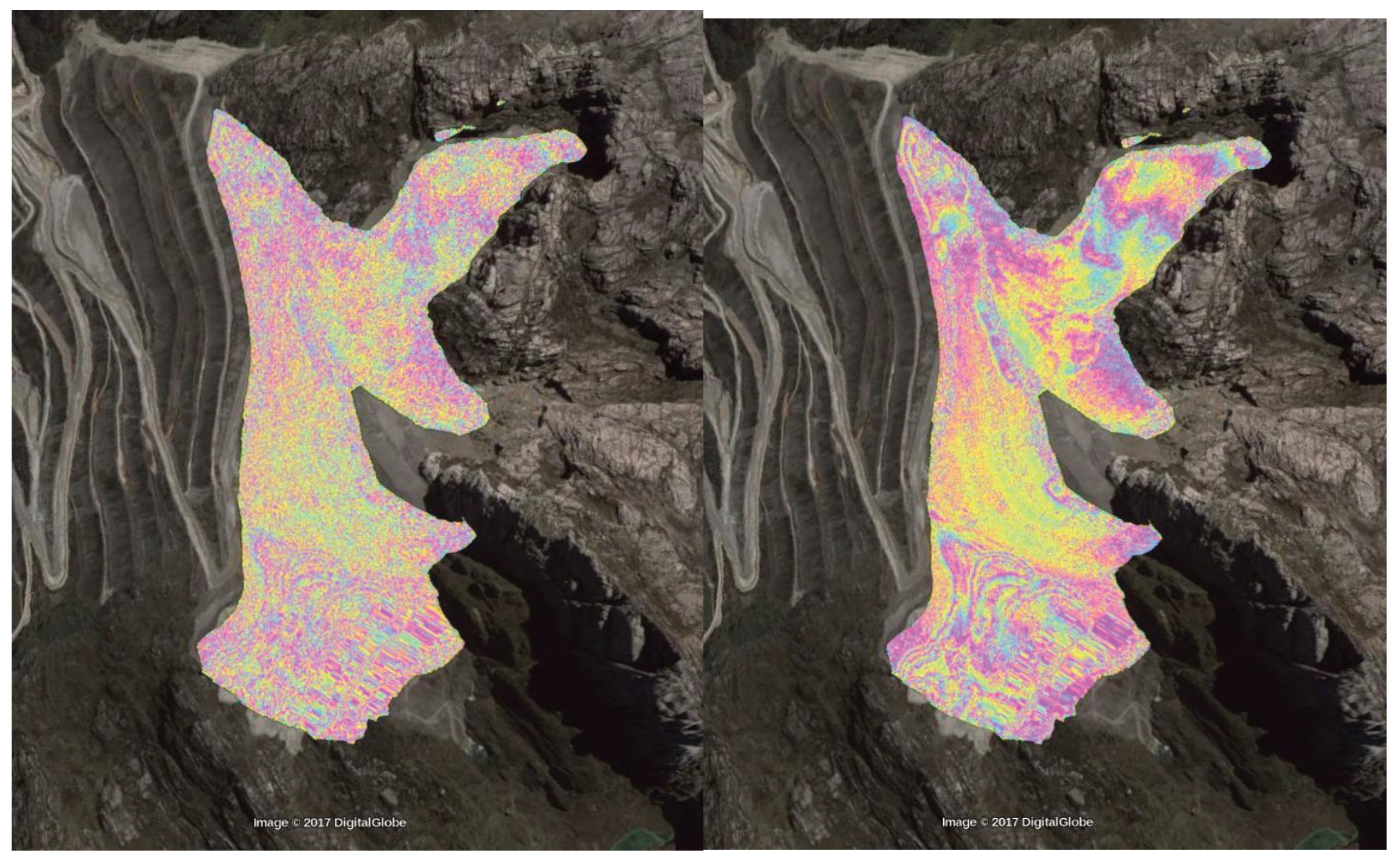

(a)

(b)

Figure 3 (a) A cropped section of an interferogram, dominated by noise, formed from SAR scenes using standard coregistration; (b) The same interferogram, but with topographic effects taken into account has much less noise

The second issue caused by highly varying elevation is related to air pressure gradients (Wadge et al. 2002), this is more correctly termed hydrostatic pressure. In general, air pressure causes a shift in an interferogram's phase values. Because hydrostatic air pressure changes with altitude, the amount of phase delay is not a constant in the interferogram and instead correlates with height. The effect at PTFI is particularly pronounced, not only because of the mountainous terrain, but also because of the size and depth of the pit, which creates a hydrostatic pressure well. 
The strategy incorporated at PTFI to mitigate this effect, is to fit a model of phase changes versus DEM heights, for each interferogram. These models can then be subtracted from source interferograms.

Figure 4 shows an interferogram prior to hydrostatic correction (top left), the derived model (top right and bottom right), and the resulting interferogram (bottom left).

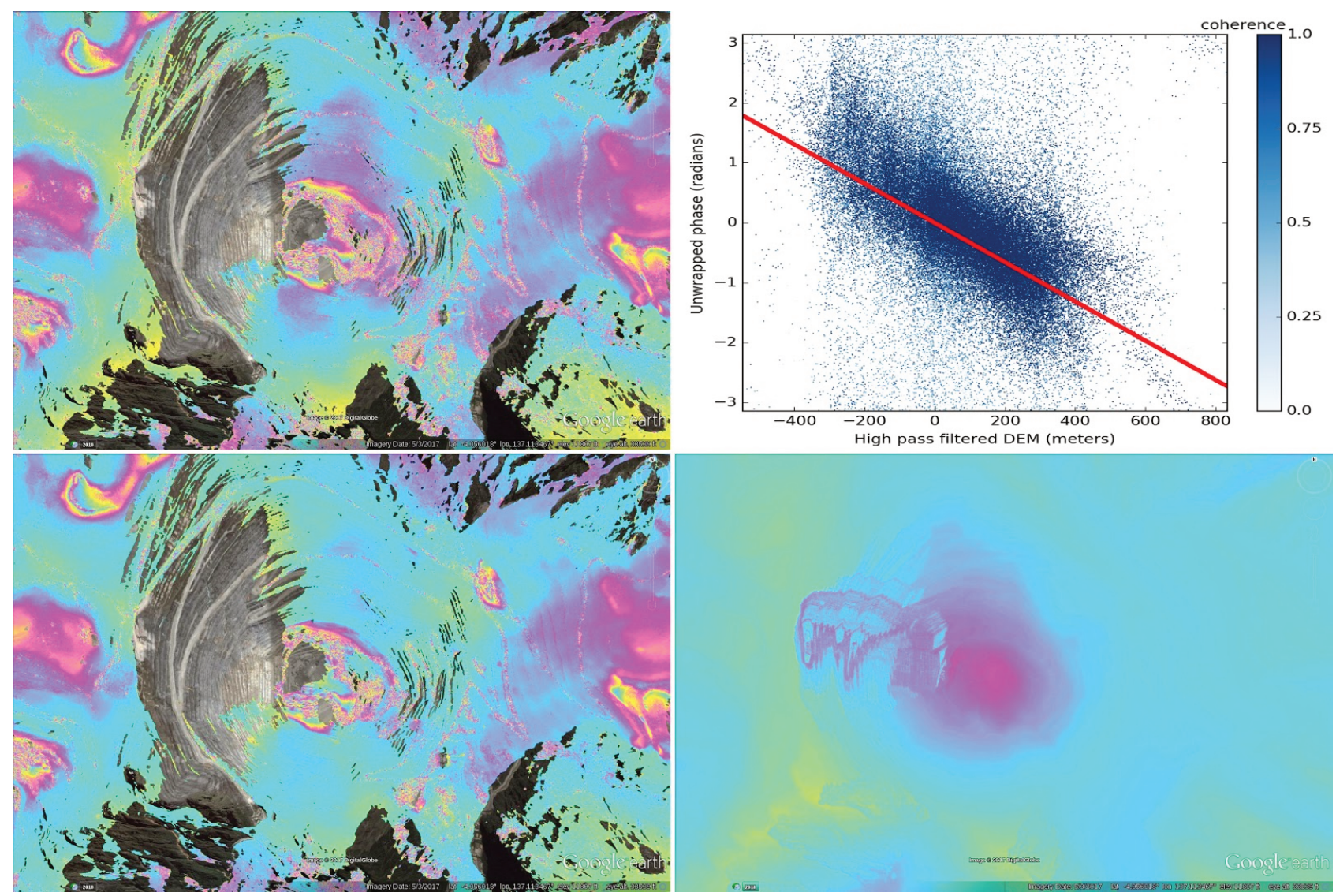

Figure 4 Top left: The hydrostatic pressure well in Grasberg pit causes spurious InSAR signals which can masquerade as ground displacement, or mask real ground displacement. Top right: A linear model is fitted to the InSAR phase versus the DEM. Bottom right: a phase screen is derived from the model. Bottom left: The screen is subtracted from the source interferogram

\subsubsection{High displacement magnitudes}

Block caving activity in the area of the DOZ causes high magnitude displacement at the surface, which in turn causes aliasing of the InSAR phase. Aliasing does not result from the absolute magnitude of the displacement being too high, but rather the spatial gradient of the displacement exceeding the wavelength of the satellite over short distances. The aliasing can be conceptualised as contour lines of displacement which densify to the point where they lie on top of one another.

Figure 5 (top) shows InSAR data over the DOZ formed from images between the 14 and 25 January 2017, the optical image on the right is shown for reference. The coloured lines that cycle through blue - pink yellow - blue are called InSAR fringes, each fringe represents $1.5 \mathrm{~cm}$ of displacement. The noise surrounding the fringes is caused by aliased displacement. 

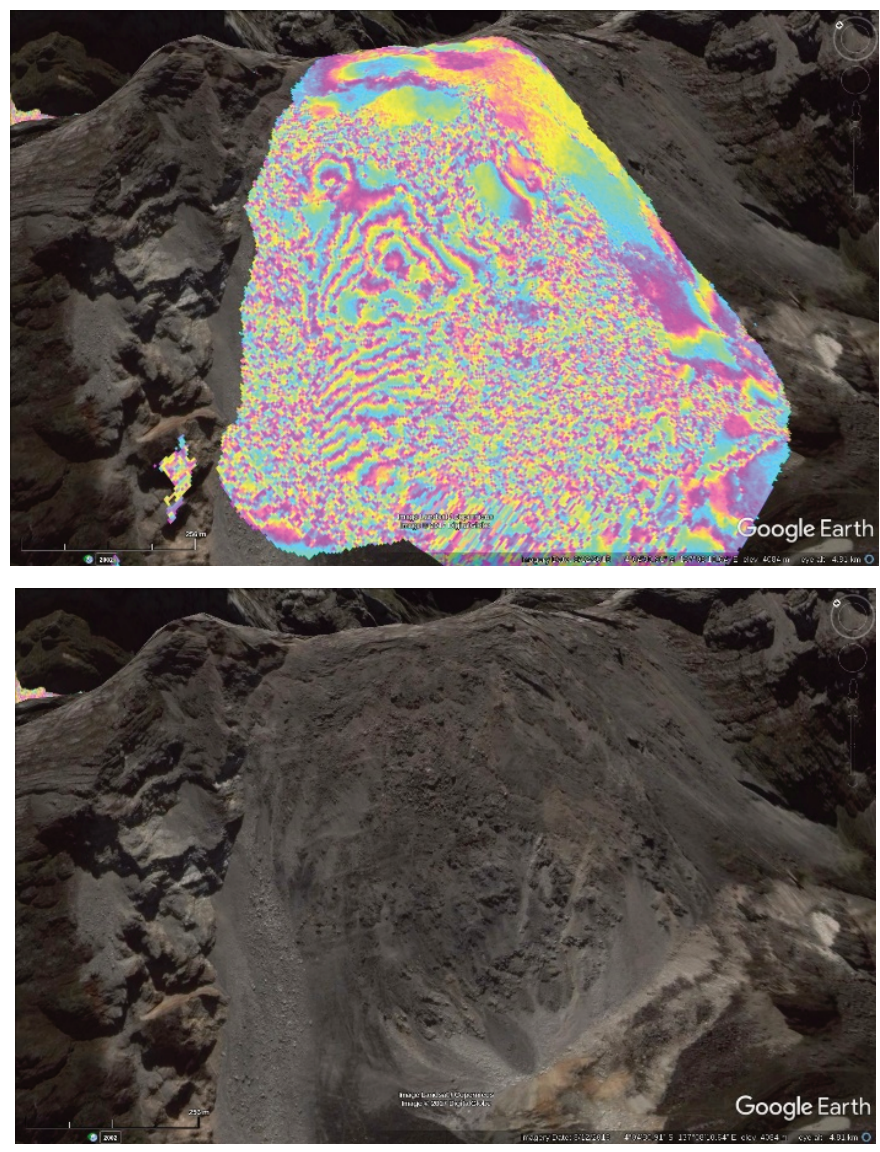

Figure 5 Top: InSAR data covering the DOZ is heavily aliased, caused by the spatial gradient of the displacement exceeding the SAR wavelength over short distances

The solution for monitoring the DOZ lies in using the amplitude of the SAR data, rather than the phase. This is readily available information and requires no extra data source. The technique is known as Speckle Tracking (Scheiber et al. 2015), and succeeds by measuring the local pixel offsets in a series of amplitude images. This is analogous to tracking motion in a series of photographs.

The precision depends on the size of the pixel, 1/20th to $1 / 10$ th of a pixel can be achieved. This is around $15-30 \mathrm{~cm}$ for TerraSAR- $X$, which is around two orders of magnitude less precise than InSAR measurements. Additionally, the resolution of the speckle tracking displacement estimates is lower than the InSAR because the correlations between images must be averaged over large areas. Improvements in terms of resolution and precision have been made by applying the semi-global matching algorithm commonly used in stereo vision.

Speckle Tracking results for the DOZ are shown in the next section.

\section{$4 \quad$ Results}

Results at PTFI include quick turnaround products, designed to provide timely alerts to new and ongoing hazards seen in a single interferogram, or as more detailed analyses involving a set of interferograms to derive displacement rate and time series information.

\subsection{Near real-time products}

Figure 6 shows a section of a mock pdf report, which depicts InSAR displacement estimated over an 11-day period, as colour-coded contours. Speckle Tracking results over the DOZ are shown as red heat map layers. These are shown in a map frame against the backdrop of a recent optical image of the site. 


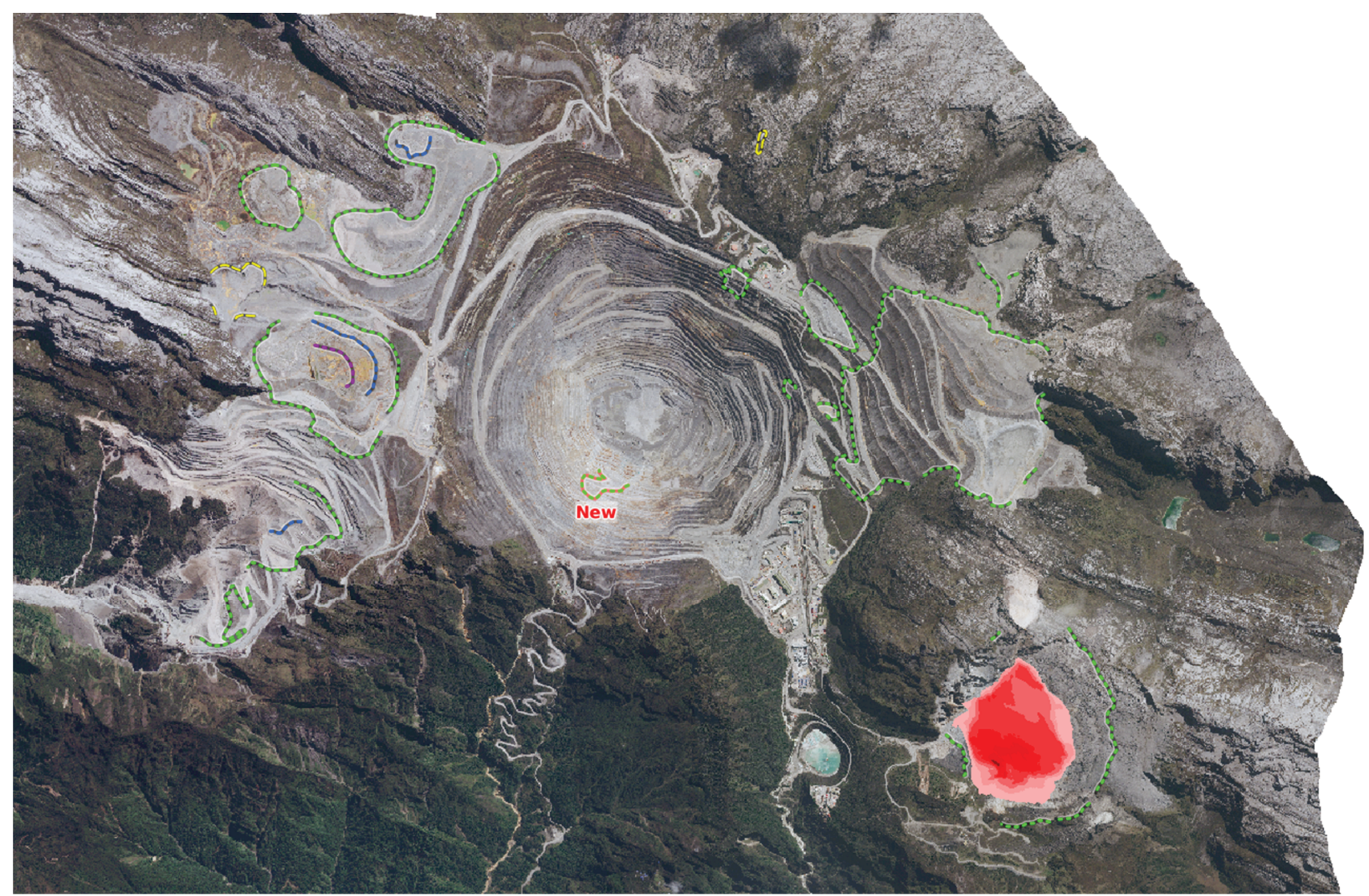

Figure 6 Section of a near real-time report covering Grasberg Mine. InSAR Displacement is shown as colour-coded contours, and Speckle Tracking as an intensifying red heat map

\subsection{Time series analysis}

A set of SAR images provide the opportunity to derive a linear displacement rate, as well as non-linear displacement time series. These are designed to append to previous analyses, providing the means to analyse results for the entire InSAR history if needed. Because Grasberg mine is captured from two different perspectives, combining the two sets of results provides a unified analysis in which the direction of displacement can be resolved for overlapping areas with sufficient displacement.

Figure 7 shows a rate from the ascending data (top left: satellite heading north, looking east), the descending data (top right: satellite heading south, looking west) and the rate for the fused dual-look results (bottom). Each pixel in the combined results is projected either to a solved direction in the up-down-east-west plane, or vertically. The increase in coverage that results from this approach is a major benefit for Grasberg, which has many steep slopes that are only visible from one of the two viewpoints. 

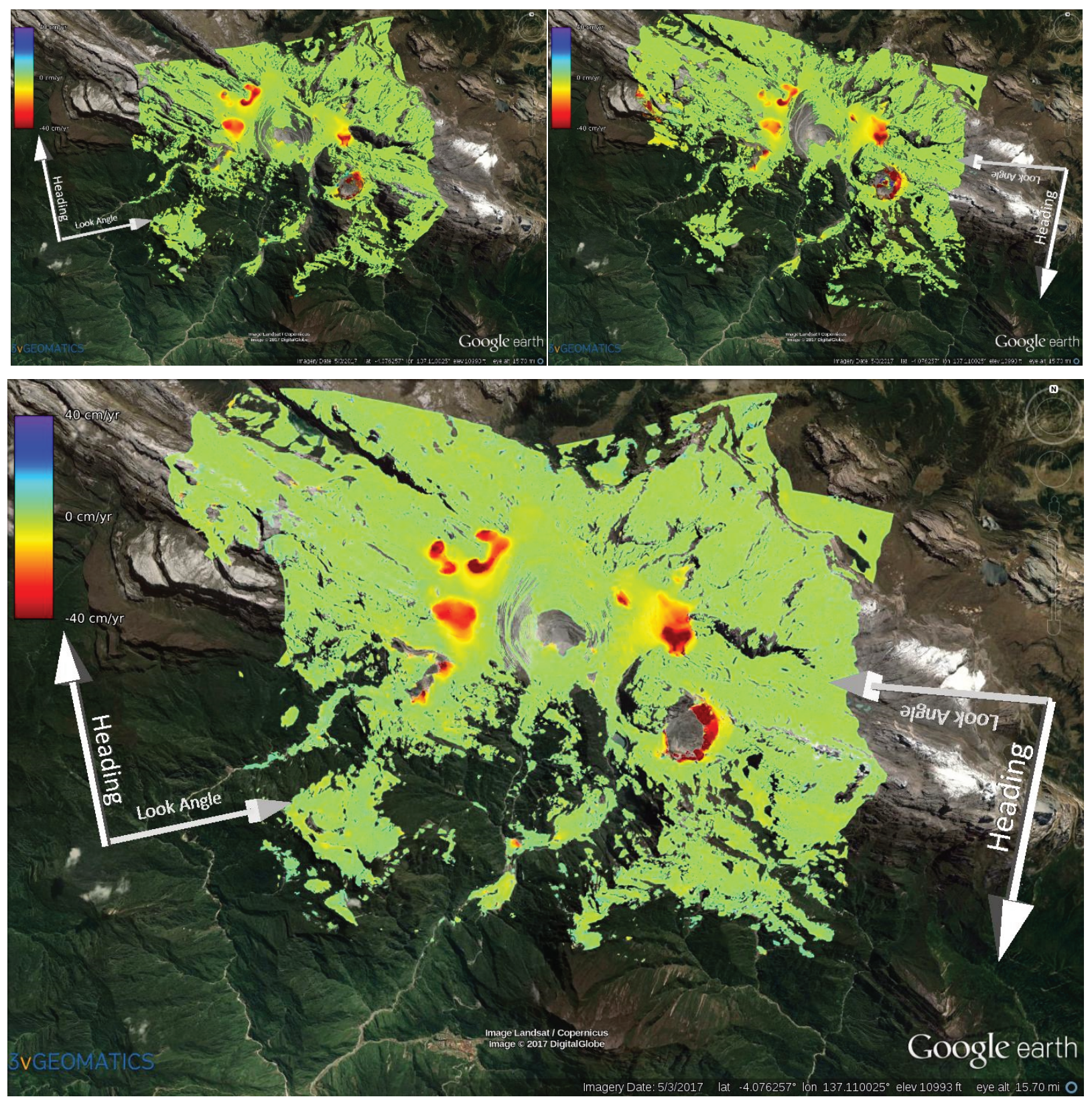

Figure 7 The ascending displacement rate (top left), the descending displacement rate (top right) and the amalgamated rate (bottom), which benefit from the increased coverage and solvable displacement directions

As well as a linear displacement rate, non-linear time series information is retrievable for every measurable pixel, which number in excess of several million for Grasberg. To allow the end user to fully utilise this large body of results, they are delivered in more than one format, including a fully enabled geospatial database. Geotech users familiar with geographic information systems (GIS) can manipulate the database to perform displacement history assessments based on arbitrarily complex selection criteria. Figure 8 is an example of a time series analysis for such a group of points. 


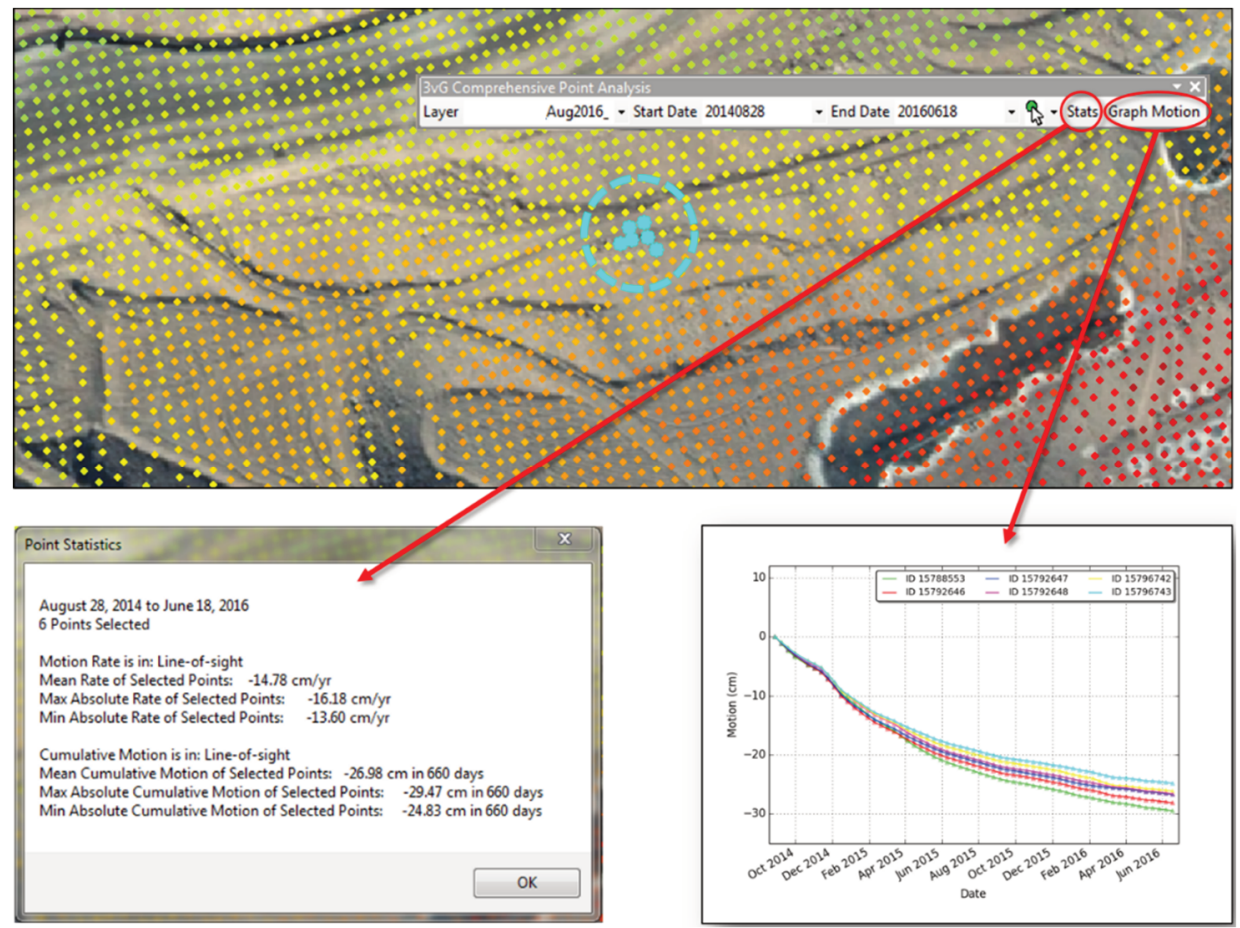

Figure 8 Typically, time series information is computed for several million pixels. GIS users can access this using a database to perform arbitrarily complex queries. Databases can easily by joined with previous databases, providing analysis of the entire InSAR history if need be

Rate and time series information can also be derived for Speckle Tracking results, covering the DOZ. Speckle Tracking displacement can be resolved in two dimensions, in the flight direction or perpendicular to it. If assumptions are made about the real direction of displacement, such as along the fall line and emergent (at right angles) to it, results can be projected to these vectors to provide a more intuitive set of results.

Figure 9 shows an example of Speckle Tracking results which have been projected to the fall line direction, where the fall line is derived for each pixel using the DEM.

Vector information can be overlaid, as shown in Figure 10. This shows the DEM derived fall line directions on the DOZ.

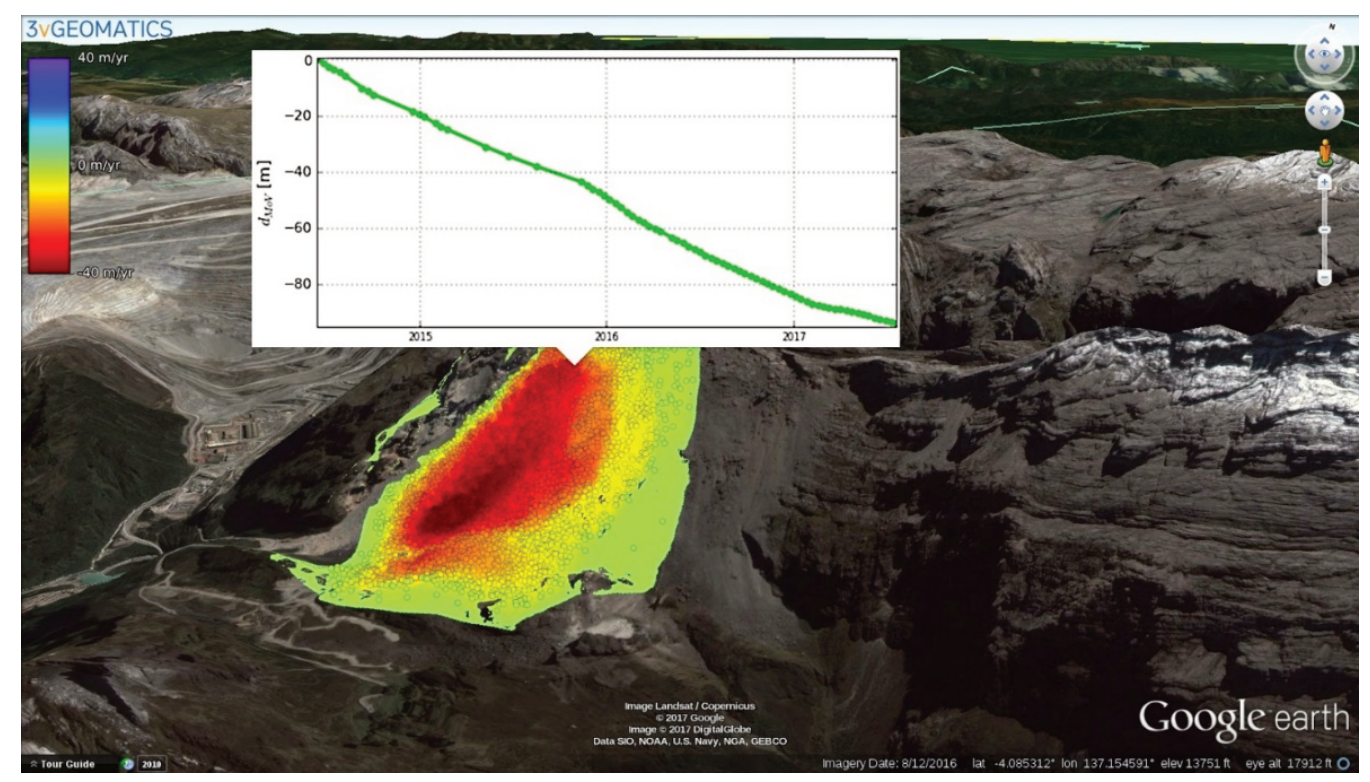

Figure 9 Displacement rate and time series information over the Deep Ore Zone, derived using amplitude speckle tracking. Displacement vectors have been reprojected along the fall line 


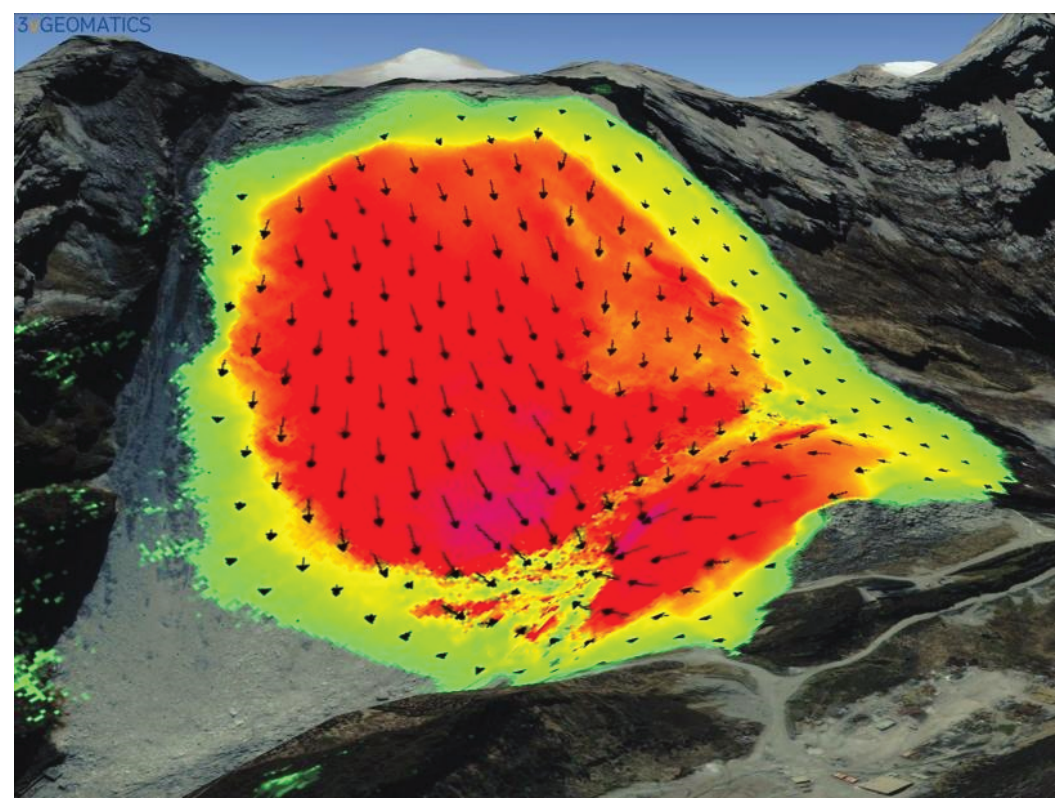

Figure 10 Arrows overlaid onto speckle tracking results show the fall line directions, derived from the digital elevation model

\section{$5 \quad$ Using InSAR results}

InSAR results at mines (and tailings storage facilities) are commonly used for detecting the onset of small subtle ground displacement. At Grasberg, the development of new and existing displacement is followed using so called 'Rapid Reports', delivered every few days, following the acquisition of each new image. These indicate displacement of around $0.5 \mathrm{~cm}$ or more, that has occurred since the previous image. These snapshot analyses are usually followed up with deeper analysis every few months to better quantify the evolution of displacement over time. Due to the data quantities involved, these 'Comprehensive Reports' are able to detect subtler displacement than Rapid Reports, on the order of 1-3 mm/a.

Following the receipt of either style of report, site teams will typically follow up any areas involving risk with a site inspection. Deployment of ground-based monitoring equipment may be required to provide more data to better understand the characteristics of any displacement. For small areas of displacement only a few metres wide, visible signs of instability may not be present, further justifying the need for ground instrumentation. InSAR at Grasberg is therefore considered complimentary to other instrumentation, rather than a replacement for it, and this is true of most operational sites employing InSAR.

Geotechnical teams at Grasberg summarise stability issues in a periodic consolidated report, making reference to InSAR results, ground inspections and ground instrumentation data. These aim to gather all the available information for distribution and to provide a means for informing decisions.

\section{Conclusion}

InSAR is an established technology for risk management at open pit and underground mines. PTFI presents a unique set of challenges for the technology, which nevertheless can be overcome through the development of tailor-made algorithms. Three specific challenges have been identified and discussed, along with the solutions to those challenges, the subsequent results and their use. 


\section{References}

European Space Agency 2014, Geometry Glossary, viewed 27 September 2017, https://earth.esa.int/handbooks/asar/CNTR5-5.html Hooper, A, Zebker, H, Segall, P \& Kampes, B 2004, 'A new method for measuring deformation on volcanoes and other non-urban areas using InSAR persistent scatterers', Geophysical Research Letters, vol. 31, L23611, https://dx.doi.org/10.1029/ 2004GL021737

Massonnet, D, Rossi, M, Carmona, C, Adagna, F, Peltzer, G, Feigl, K \& Rabaute, T 1993, 'The displacement field of the Landers earthquake mapped by radar interferometry', Nature, vol. 364, no. 8, pp. 138-142.

Nitti, DO, Hanssen, KF, Refice, RF, Bovenga, A \& Nutricato, F 2011, 'Impact of DEM-assisted coregistration on high-resolution SAR interferometry', IEEE Transactions on Geoscience and Remote Sensing, vol. 49, no. 3, pp. 1127-1143.

Scheiber, R, Jager, M, Prats-Iraola, P, De Zan, F \& Geudtner, D 2015, 'Speckle tracking and interferometric processing of TerraSAR-X TOPS data for mapping nonstationary scenarios', IEEE Journal of Selected Topics in Applied Earth Observations and Remote Sensing, vol. 8, no. 4, pp. 1709-1720, https://dx.doi.org/10.1109/jstars.2014.2360237

Wadge, G, Webley, PW, James, IN, Bingley, R, Dodson, A, Waugh, S, ... \& Clarke, PJ 2002, 'Atmospheric models, GPS and InSAR measurements of the tropospheric water vapour field over mount etna', Geophysical Research Letters, vol. 29, no. 19, pp. 11-1-11-4. 
